\title{
The NLC Photon Collider Option Progress and Plans
}

\author{
J. Gronberg
}

This article was submitted to International Workshop on High Energy Photon Colliders, Hamburg, Germany, June 14-17, 2000

\section{August 31, 2000}

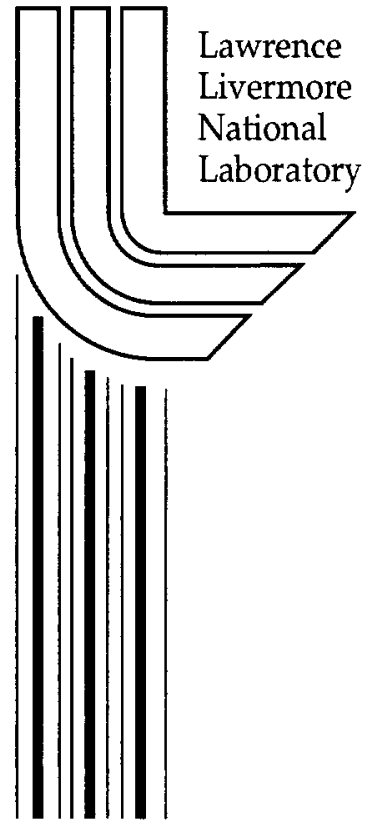




\section{DISCLAIMER}

This document was prepared as an account of work sponsored by an agency of the United States Government. Neither the United States Government nor the University of California nor any of their employees, makes any warranty, express or implied, or assumes any legal liability or responsibility for the accuracy, completeness, or usefulness of any information, apparatus, product, or process disclosed, or represents that its use would not infringe privately owned rights. Reference herein to any specific commercial product, process, or service by trade name, trademark, manufacturer, or otherwise, does not necessarily constitute or imply its endorsement, recommendation, or favoring by the United States Government or the University of California. The views and opinions of authors expressed herein do not necessarily state or reflect those of the United States Government or the University of California, and shall not be used for advertising or product endorsement purposes.

This is a preprint of a paper intended for publication in a journal or proceedings. Since changes may be made before publication, this preprint is made available with the understanding that it will not be cited or reproduced without the permission of the author.

This work was performed under the auspices of the United States Department of Energy by the University of California, Lawrence Livermore National Laboratory under contract No. W-7405-Eng-48.

This report has been reproduced directly from the best available copy.

Available electronically at http://www.doc.gov/bridge

Available for a processing fee to U.S. Department of Energy

And its contractors in paper from

U.S. Department of Energy

Office of Scientific and Technical Information

P.O. Box 62

Oak Ridge, TN 37831-0062

Telephone: (865) 576-8401

Facsimile: (865) 576-5728

E-mail: reports@adonis.osti.gov

Available for the sale to the public from

U.S. Department of Commerce

National Technical Information Service

5285 Port Royal Road

Springfield, VA 22161

Telephone: (800) 553-6847

Facsimile: (703) 605-6900

E-mail: orders@ntis.fedworld.gov

Online ordering: http://www.ntis.gov/ordering.htm

\section{OR}

Lawrence Livermore National Laboratory

Technical Information Department's Digital Library

http://www.llnl.gov/tid/Library.html 


\title{
The NLC Photon Collider Option Progress and Plans
}

\author{
Jeffrey Gronberg à \\ ${ }^{a}$ Lawrence Livermore National Laboratory \\ 7000 East Ave. Livermore, CA 94550
}

\begin{abstract}
The idea of producing beams of high energy photons by compton backscattering of laser photons was proposed over 20 years ago. At the time, producing the required laser pulses was not feasible. However, recent advances in high average power, diode pumped lasers appear to have solved this problem. The US Collaboration is now turning its attention to the engineering requirement of mating the laser and optics components with the accelerator structures in the confined space of the a colliding beam interaction region. The demonstration of a technically feasible interaction region design is planned for the Snowmass conference in 2001.
\end{abstract}

Key words: HEP; Gamma-Gamma Collider

\section{Introduction}

One of the options being considered for the next generation of high energy physics machines is a linear $e^{+} e^{-}$collider at $500 \mathrm{GeV}$ center of mass energy (1). This opens up the possibility of producing gamma-gamma collisions at high energy by compton backscattering of low energy photons off of the high energy electron beam (2). Gamma-gamma collisions provide a unique window on new physics through the Higg's boson productions process $\gamma \gamma \rightarrow H^{0}$. Since this process proceeds through a loop diagram in which all particles with charge and mass contribute, it is sensitive to the existence of new particles with masses up to the Plank scale (3).

The first gamma-gamma collider studies showed the need for lasers that were beyond the state of the art in laser technology. To maximize the gammagamma luminosity a laser is required which produces pulses of 1 picosecond duration to match the longitudinal length of the electron bunches. They also need to produce 1 Joule per pulse to maximize the number of backscattered 
gamma ravs. And, finally, the laser must have enough average power to produce $\sim 11000$ pulses per second.

The problem of producing picosecond pulses was solved with the development of chirped pulse amplification. which reduced pulse duration from nanoseconds to picoseconds. Lasers that could produce pulses of 1 Joule in 1 picosecond became commonplace in laboratories. However, they typically functioned with a low duty cycle and with low power conversion efficiency. In studying the properties of these lasers it was noticed that unlike nanosecond pulses, which tend to melt and vaporise a target, picosecond pulses have electric fields that are intense enough to disintegrate matter, leaving a very clean edge (5). This created a great interest in increasing the average power of these lasers so that they could be used for industrial applications. The current state of the art in laser technology has reached a point where a gamma-gamma collider is now in the realm of possibility.

The following sections contain a review of the various components required to create gamma-gamma collisions and a description of the work that remains to produce a unified and feasible design.

\section{Status of Enabling Technologies}

Starting from the basic NLC design for an $e^{+} e^{-}$collider, the production of gamma-gamma collisions requires the addition of a laser subsystem. The laser pulses must be produced, formatted to match the time structure of the electron beam, transported to the interaction region, focused to collide with the electron beams within a few millimeters of the interaction point, and the unused portion of the laser pulse extracted.

Lasers that can produce a pulse of one Joule in a picosecond have existed for a decade. However. a gamma-gamma collider must produce a laser pulse for every electron bunch to maximize the gamma-gamma luminosity. Since there are 120 trains per second, each having 95 bunches, 11,400 pulses per second will need to be produced. This would require an average laser power of 11.4 kilowatts. Nominally, we would need to multiply this number by two to provide pulses for both beams, however, clever focusing schemes allow a single pulse to be used for one bunch in each beam.

Ti:Sapphire lasers are curently capable of generating 10 Watts of average power. Since they were designed as lasers that pump other lasers they have poor power conversion efficiency. The typical ratio of delivered laser power to wall plug power is $10^{-5}$ and so a system based on these lasers would require a thousand laser tables and have a total system power requirement of 1 gigawatt. 


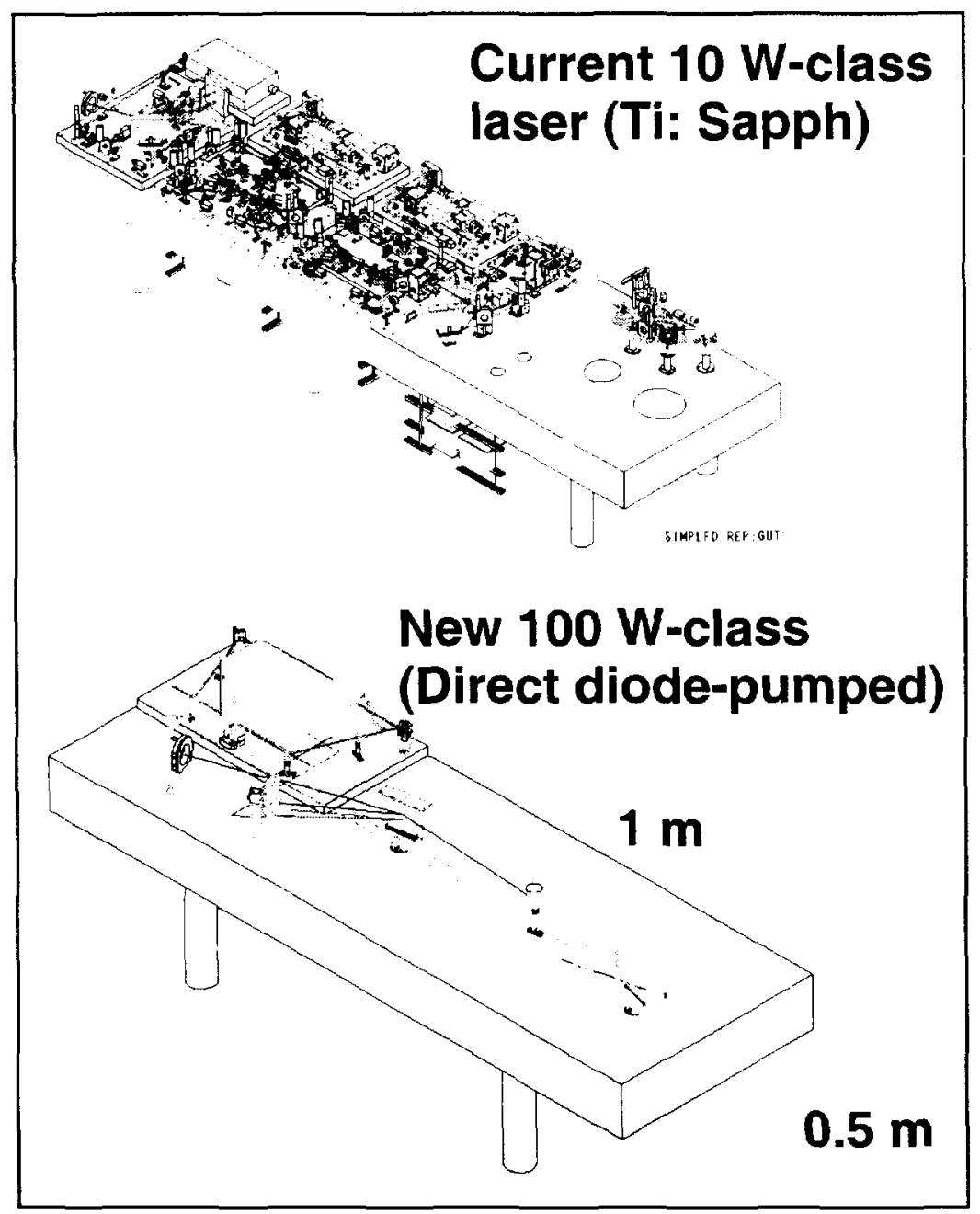

Fig. 1. A comparison of the laser table layout between Ti:Sapphire and diode pumped lasers. Diode pumped lasers have a clear reduction in complexity.

this is quite impractical for the needs of a gamma-gamma collider. These systems are also quite complicated, as can be seen in figure 1.

In contrast, the new technology of glass diode pumped lasers solves most of these problems. Since the light output of the diodes can be tuned to the pump frequency of the laser they are very efficient at converting wall plug power to laser light. Efficiencies of $5-10 \%$ can be achieved, leading to total power requirements of only $200 \mathrm{~kW}$ for the entire laser system. Current designs are capable of producing 40 Watts of average power with designs capable of 100 Watt average power under development. This would mean that only 95 laser tables would be needed to produce the required pulses. The reduced complexity of the laser table, see figure 1, combined with the need for fewer tables would lead to a reduced capital cost for the laser plant.

One of the driving costs for a glass diode pumped laser in previous estimates was the cost of the diodes. Sufficient peak diode power must be supplied to 

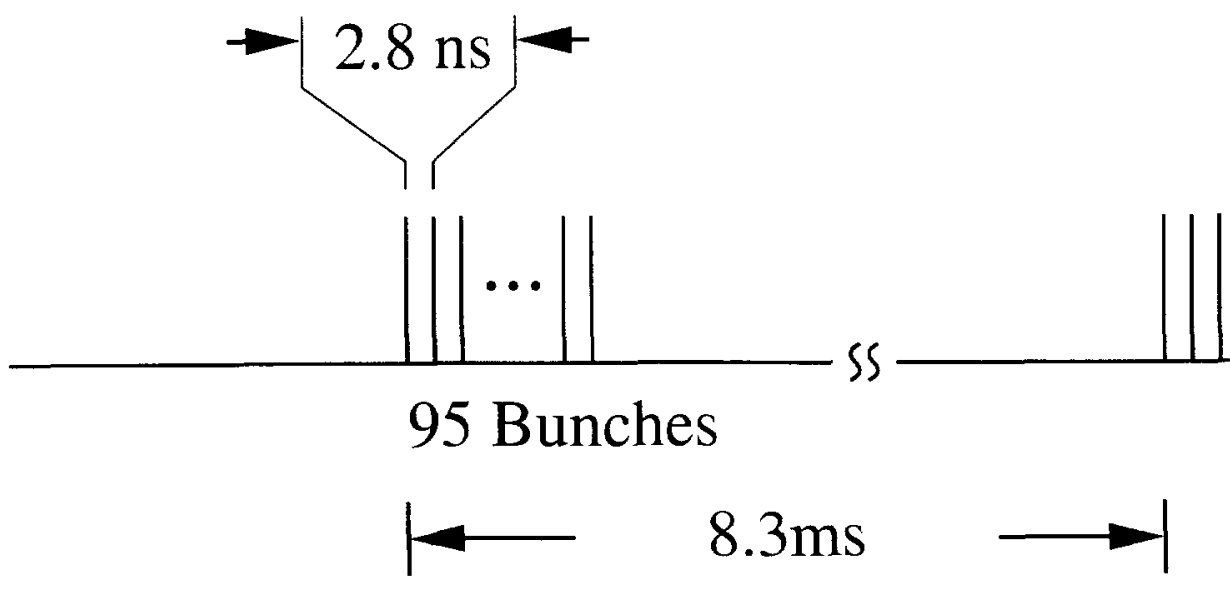

\section{Pdiode}

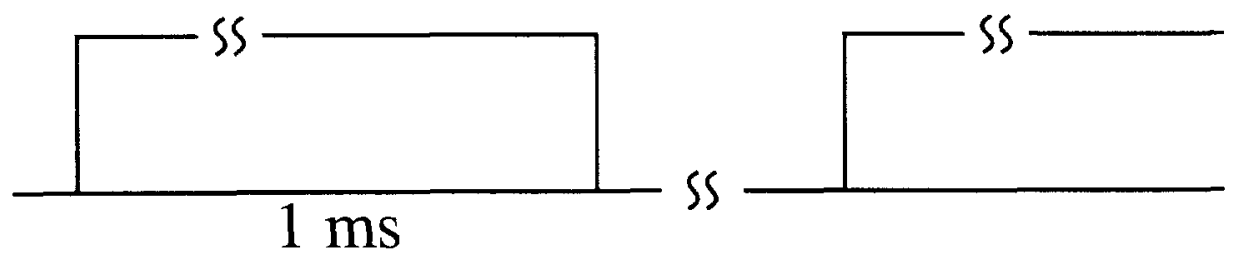

Fig. 2. The time structure of bunches in the NLC is shown on the top line with a comparision to the upper state lifetime of the glass laser medium.

populate the upper state of the laser in a time comparable to the upper state lifetime. The new laser materials have a rather long upper state lifetime of 1 millisecond, which reduces the diode cost. As shown in figure 2, an upper state lifetime of one millisecond can easily fit between the bunch trains but not between individual bunches. Therefore enough energy for 95 pulses of 1 Joule need to be transfered to the laser medium within one millisecond. Equation 1 shows the calculation of the peak diode power required, assuming $25 \%$ efficiency for converting diode power into laser light, $\epsilon_{\text {opt-opt }}$, and conservatively including a factor of 2 for the two beams. We find,

$$
P_{\text {diode }}^{\max }=\frac{2.0 \times N_{\text {bunch }} \times E_{\text {bunch }}}{\epsilon_{\text {opt-opt }} \times \tau_{u}}=\frac{2.0 \times 95 \times 1.0 \mathrm{~J}}{0.25 \times 10^{-3} \mathrm{sec}}=0.76 \mathrm{MW} .
$$

The figure of 0.76 megawatts is reduced compared to previous estimates due to the long upper state lifetime. Since diodes can be produced today for about $\$ 5$ per watt, this leads to a total diode cost for the system of 3.5 million dollars, removing it as the dominant cost of the project.

Once the laser pulses are produced they must also be combined and formatted in time to match the structure of the NLC bunch trains. As can be seen in figure 2, individual bunches are separated 2.8 nanoseconds while the trains of 95 bunches are separated by 8.3 milliseconds. The lasers group at LLNL has developed a concept to combine individual pulses into a pulse train using 


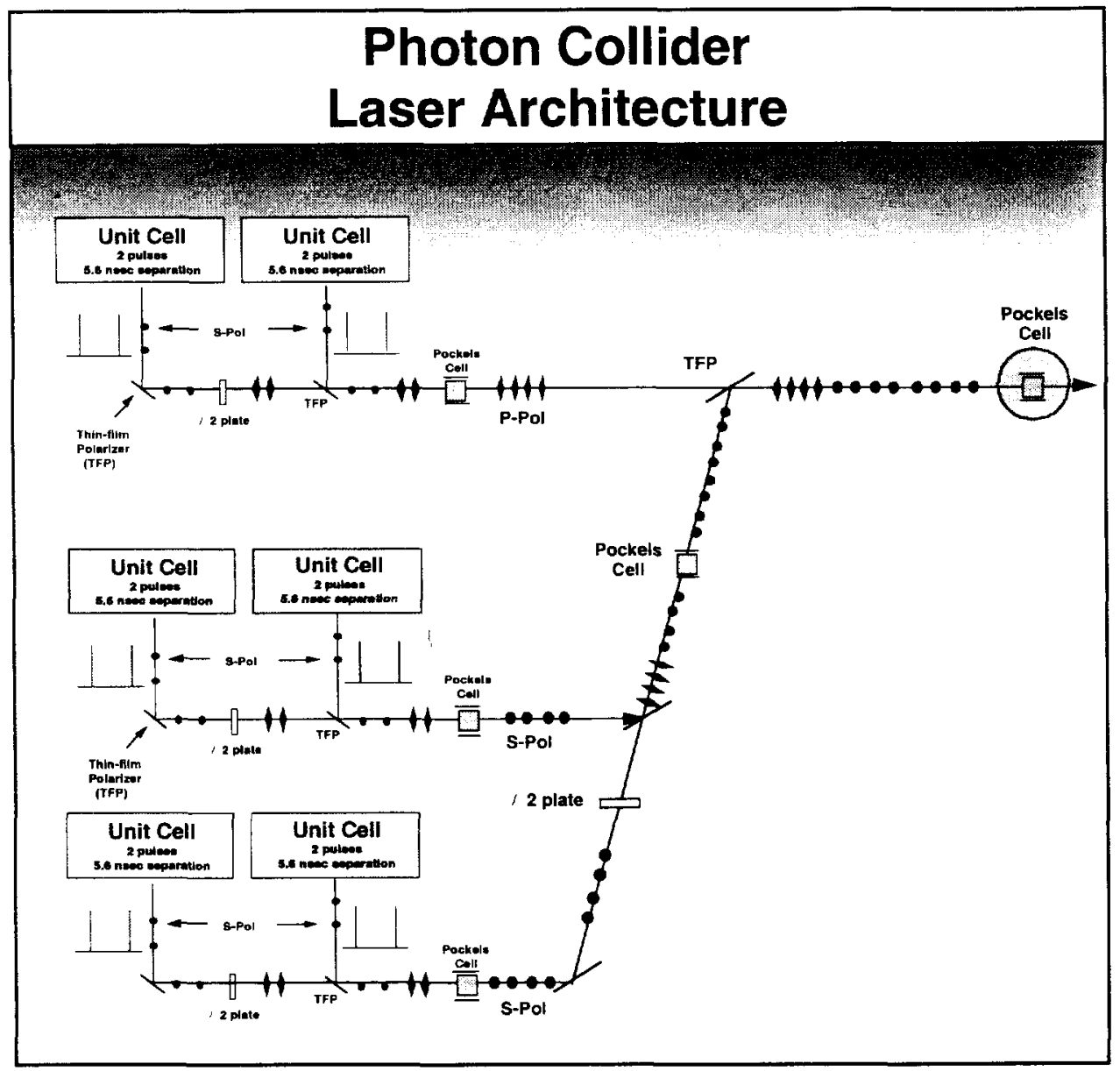

Fig. 3. A schematic of the proposed system for assembling the output of individual laser tables into a pulse train.

thin film polarizers, half-wave plates and pockels cells. A schematic of the system is shown in Figure 3. A thin film polarizer allows pulses from different beam lines, with different polarizations, to be combined into a single beam. Once combined, both half-wave plates and pockels cells are used to change the polarization of the pulses in preparation for the next combining stage. The pockels cells must be fast enough to change state in the 2.8 nanosecond window between pulses. All of the components required are available today except for the final pockels cell, which must be able to handle the maximum average power. Viable concepts for a high average power pockels cell exist but they remain to be developed and demonstrated.

Finally, the pulse trains must be compressed down to the picosecond scale, directed into the interaction region, and focused down to a point for collision with the electron bunch. An initial design of the optics necessary to focus the laser pulses was presented at the Snowmass conference in 1996 (4). The high power of the laser pulses and the need to maximize the gamma-gamma luminosity drives several design choices. Transmissive optics had to be ruled 


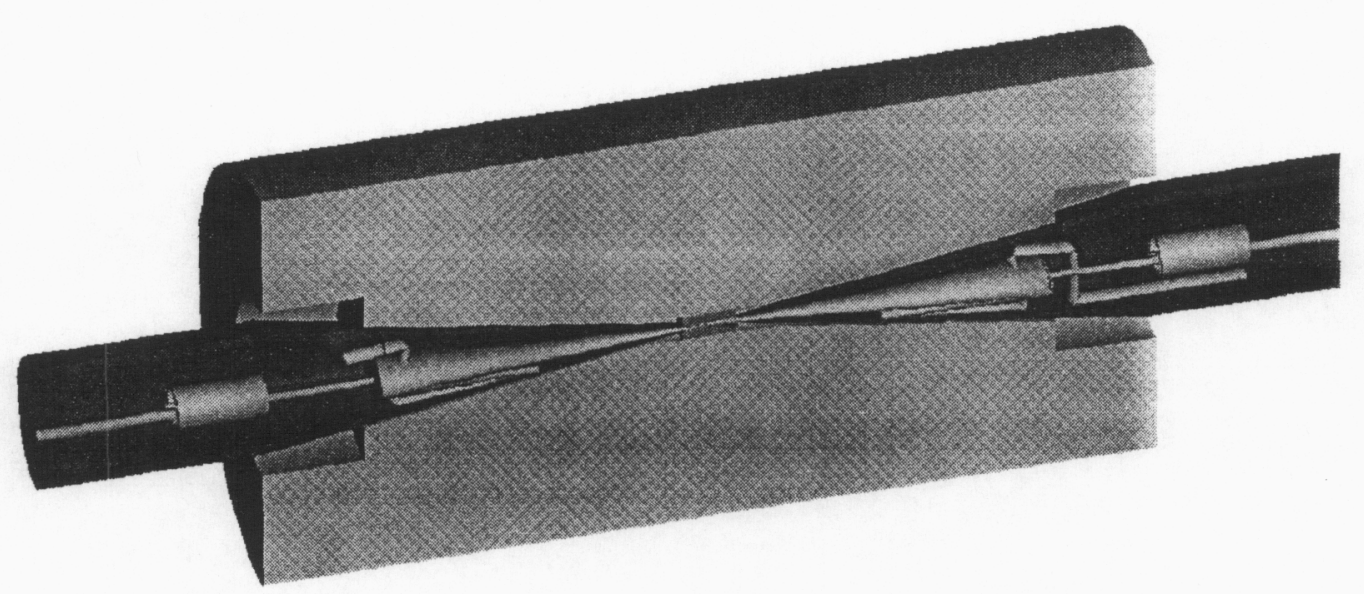

Fig. 4. A view of the interaction region showing the available space for the laser focusing optics. Laser pulses enter from the right and are focused down to a spot a few millimeters from the interaction point (IP). The pulses then enter the telescope on the left side, are expanded, reflected, and refocused onto the other beam a few millimeters from the IP.

out due to the non-linearities in the index of refraction for high power pulses. Instead, a four mirror telescope design, using all reflective components was chosen. Figure 4 shows the optics elements in the space allowed by the detector. The focal length is chosen to keep the laser intensity at a level that avoids causing non-linear effects in the compton backscattering. Also, the final focusing mirror must be on-axis with the beam, since the compton backscattering process becomes less efficient if there is an angle between the laser pulses and the electron beam. After the laser pulse passes through the bunch, it is then collected by a complementary four mirror telescope on the other side of the interaction region, reflected, and refocused onto the opposite beam. This reduces the needed laser power by almost a factor of two.

\section{$3 \quad$ Integration Issues}

While there has been great progress on many of the individual components necessary for a gamma-gamma collider, it still needs to be demonstrated that 
these components can be integrated with the accelerator and detector in a real interaction region. The NLC management has asked the US gamma-gamma working group to demonstrate a feasible design of a gamma-gamma interaction region by the time of the Snowmass conference in 2001. A multi-disciplinary team that includes laser experts, optical designers, accelerator physicists and high energy experimentalists has been assembled to address this challenge.

The team will need to address a wide range of issues. A specific choice for the laser technology must be made and developed. A decision on how to mount, stabilize, cool and align the optics will be required. The radiation levels that the optics will need to endure must be simulated along with a calculation of the heating of the optics from the electron beam wakefields. The final focus system for the accelerator will be studied to determine its potential for round beam operation, which can increase luminosity. Disruption of the electron beam by the dielectric material of the optics will have to be evaluated. The design of the masking and outgoing beam dump lines will need to be studied to prevent beam-beam interaction particles and spent beam particles from creating backgrounds for the detector which could limit the physics reach. Finally, the tradeoffs between various effects will have to be studied to optimize the physics performance of the final design.

The goal of this effort is to deliver a detailed technical report, suitable for review, for the Snowmass conference in 2001 . The report will include the facilities requirement documents, a detailed work breakdown structure including cost and scheduling information, a complete and integrated design of a gammagamma interaction region, and a layout of a second collider hall capable of accomadating the additional laser subsystem.

\section{Conclusions}

The progress in high average power laser technology affords the field of high energy physics a new opportunity to study a heretofore inaccessible class of particle collisions, requiring only a small addition to the cost of an $e^{+} e^{-}$linear collider. While the basic technology seems ready, a complete mechanical design with an accurate estimate of physics reach is required inorder for the HEP community to make informed decisions about future projects. The US Gamma-Gamma working group has committed itself to providing this study and making it available for the Snowmass conference in June, 2001. 


\section{Acknowledgments}

This work was performed under the auspices of the U.S. Department of Energy by the Lawrence Livermore National Laboratory under Contract No. W-7405Eng-48.

\section{References}

[1] The NLC Design Group, "Zeroth Order Design Report for the Next Linear Collider", LBNL-5424, SLAC-474, UCRL-ID-124161, UC-414 (1996).

[2] V. Telnov, Nucl. Instr. and Meth. A 355 (1995) 3.

[3] J. Ellis, M.K. Gaillard and D. Nanaopoulos, Nucl. Phys. B 106 (1976) 292; M. Shifman et al., Sov. J. Nocl. Phys. 30 (1979) 711.

[4] The Gamma-Gamma Working Group, "A Second Interaction Region For Gamma-Gamma, Gamma-Electron \& Electron-Electron Collisions for NLC", LBNL-38985, LLNL-UCRL-ID 124182 SLAC-PUB-95-7192 (1996).

[5] M.D. Perry and G. Mourou, Science 264 (1994) 917. 